\title{
Performance of the Panbio COVID-19 Rapid Antigen Test Device for infection control purposes
}

\author{
Javier Ena ${ }^{1 *}$, Carmen Martinez-Peinado ${ }^{2}$, Francisco Arjona-Zaragozi ${ }^{2}$, and José V. Segura-Heras ${ }^{3}$ \\ ${ }^{1}$ Internal Medicine Service; ${ }^{2}$ Microbiology Service. Hospital Marina Baixa, Villajoyosa; ${ }^{3}$ Centro de Investigación Operativa. Ed. Muhammad Al-Shafra, \\ Universidad Miguel Hernández, San Juan de Alicante. Alicante, Spain
}

\begin{abstract}
Objective: We assessed the performance characteristics of the Panbio COVID-19 Rapid Antigen Test Device in field conditions and its potential use as a point-of-care test for isolation and cohorting patients at risk. Methods: We reviewed our laboratory records from January 2021 to May 2021 to identify subjects with a Panbio COVID-19 Rapid Antigen Test Device (index test) and a real-time reverse transcriptase-polymerase chain reaction (RT-PCR) (reference test) test carried out on the same date. Results: In total, 634 subjects met inclusion criteria. Three $(0.2 \%)$ samples of the index test were not interpretable. A total of $51(8.08 \%)$ subjects had SARS-CoV-2 infection. The index test had sensitivity $78.85 \%$ (95\% Cl: $66.30-88.94 \%)$, specificity 91.58\% (95\% Cl: $89.02-93.71)$, and accuracy $90.54 \%$ (95\% Cl: 87.99-92.70\%). A total of 11 (1.73\%) subjects (one asymptomatic, five with respiratory symptoms, and five with pneumonia) had a false-negative result of the index test with median (range) cycle threshold RT-PCR of 25 (21-30), indicating the presence of high viral load in the nasopharyngeal sample. Conclusions: The Panbio COVID-19 Rapid Antigen Test Device cannot replace SARS-CoV-2 RT-PCR for infection control purposes.
\end{abstract}

Key words: Coronavirus infections/^diagnosis. Sensitivity and specificity. COVID-19 testing.

\section{Introduction}

The pandemic caused by coronavirus 2 (SARS-CoV-2) continues to challenge the health systems. One of the basic strategies in controlling the pandemic is identifying cases and contacts to carry out their isolation ${ }^{1}$.

Due to its high sensitivity and specificity, the gold standard for diagnosing SARS-CoV-2 infection recommended by the WHO and FDA is detecting the virus by the real-time reverse transcriptase-polymerase chain reaction (RT-PCR $)^{2,3}$. However, RT-PCR is a laboratory procedure that requires sophisticated equipment and trained personnel to perform the test. The turnaround time varies according to geographical location and proximity to the testing laboratory. Sometimes there have been delays in completing the RT-PCR tests due to demand overload or lack of reagents.

Antigen detection tests for the diagnosis of SARSCoV-2 infection have lower diagnostic performance than the detection of the virus by RT-PCR ${ }^{4}$. However, they can be used as point-of-care tests for SARSCoV-2 disease with the results available in $15 \mathrm{~min}$. Several commercial antigen detection kits for SARSCoV-2 diagnosis are available ${ }^{5}$, and one of the most commonly used is the Panbio COVID-19 Rapid Antigen Test Device. Compared with RT-PCR, the manufacturer claimed a sensitivity of $98.1 \%$ and a specificity of $99.8 \%$ in 508 symptomatic subjects. Furthermore, during the epidemic, the Panbio COVID-19 Rapid Antigen Test

\section{Visual abstract available at https://spanishjmed.com/frame_esp.php?id=55}

\section{Corrrespondence:}

*Javier Ena

E-mail: ena_jav@gva.es

2696-5631 / (C 2021 Sociedad Española de Medicina Interna. Published by Permanyer. This is an open access article under the CC BY-NC-ND license (http://creativecommons.org/licenses/by-nc-nd/4.0/). 
Device performance reproduced manufacturer results ${ }^{6}$. However, in field conditions, the performance characteristics have not been thoroughly evaluated. Moreover, the Panbio COVID-19 Rapid Antigen Test used as a surrogate marker of SARS-CoV-2 transmission capability is unknown.

The purpose of our study was to evaluate in field conditions the diagnostic performance of the Panbio COVID-19 Rapid Antigen Test Device compared with RT-PCR for SARS-CoV-2. We also assessed the potential use of the Panbio COVID-19 rapid antigen test for infection control purposes to isolate and cohort cases and high-risk contacts.

\section{Methods}

\section{Study setting}

We carried out the study in a 280-bed hospital belonging to the National Health System (Generalitat Valenciana) that serves a registered population of 190,000 inhabitants on the east coast of Spain.

\section{Study design}

We have followed the recommendations of the STARD guideline for reporting Diagnostic Studies in preparing the document? .

We carried out a retrospective study reviewing our Clinical Microbiology Department data between January and May 2021. We selected for the study those subjects who had the Panbio COVID-19 Rapid Test Device (Abbott Abbott Diagnostic GmbH, Jena, Germany) and the RT-PCR (Genesig ${ }^{\circledR}$ COVID-19 2G Real-Time PCR Assay) carried out in nasopharyngeal samples on the same day. Trained healthcare professionals collected nasopharyngeal swabs.

\section{Population}

We included adult and pediatric populations if they had COVID-19 symptoms lasting for $<7$ days or subjects exposed to a patient with SARS-CoV-2 infection. We collected from the Clinical Microbiology Department records information about the primary complaint, results of rapid antigen test, and RT-PCR.

\section{Rapid diagnostic antigen testing}

The Panbio'TM COVID-19 Ag Rapid Test Device (index test) is a membrane-based immunochromatography assay that detects the nucleocapsid protein of SARSCoV-2 in nasopharyngeal samples ${ }^{8}$. For the procedures, we followed the manufacturer's recommendations. Tests results were recorded after $15 \mathrm{~min}$ of assay initiation by one observer. Only the control line and no test line within the result window indicated a negative result. The presence of the test line and the control line within the result window, regardless of which line appeared first, showed a positive result. The result was invalid if the control line was not visible within the result window after performing the test.

\section{RT-PCR testing}

We tested nasopharyngeal swab samples as the reference test by RT-PCR (Genesig ${ }^{\circledR}$ COVID-19 2G RealTime PCR Assay) ${ }^{9}$. This multiplex assay is a CE marked, in vitro diagnostic, real-time, RT-PCR intended for the qualitative detection of nucleic acid from SARSCoV-2 (ORF1ab and S gene targets) in nasopharyngeal swabs, oropharyngeal swabs, and sputum specimens. Amplification and detection were performed for 45 cycles on a Biorad CFX96 thermocycler (Biorad Laboratories); the manufacturer's software automatically determined the threshold cycle (Ct). A positive result was defined as amplification of any of the two SARS-CoV-2 genes. We used a cycle threshold value $<30$ to determine clinically relevant concentrations of SARS-CoV-2 since culturing of SARS-CoV-2 was not possible at Ct-values above $29^{10}$.

\section{Statistical analysis}

We expressed continuous variables as mean and standard deviation and categorical variables as frequency and percentage. Using the RT-PCR results as the reference test, we calculated the performance characteristics of the Panbio COVID-19 Rapid Antigen Test Device. All statistical analyses were performed with the R program (R 4.1.0 for Windows).

\section{Ethics statement}

The Research Commission approved the study of our center. As it was a retrospective study, the patients' informed consent was not considered necessary by the Research Commission. The treatment of personal data was governed by organic law 15/1999 and Royal Decree 1720/2007 for its protection. 


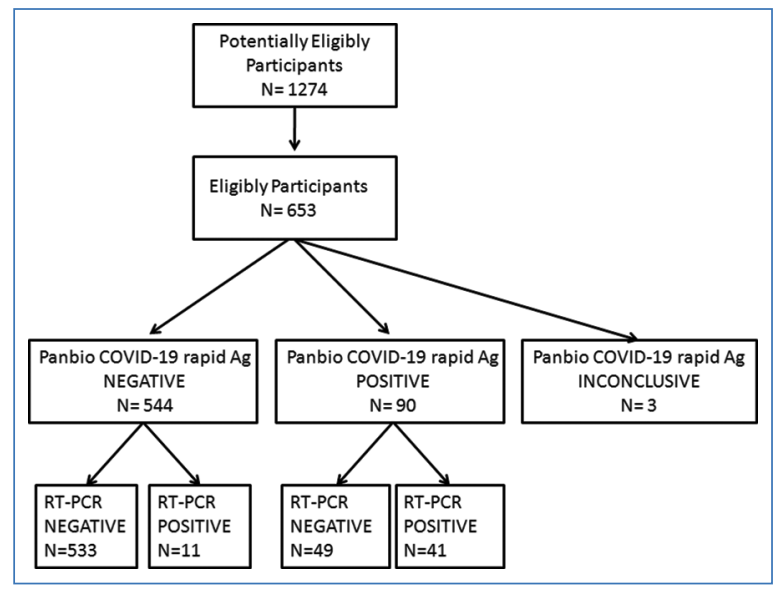

Figure 1. Patient flow chart.

\section{Results}

\section{Participants}

From a total of 1274 eligible participants with matched pairs of the index and reference test, we found $634(49.8 \%)$ subjects with enough clinical information (Fig. 1). All participants had a time interval between symptoms onset to the sampling of fewer than 7 days. Subjects' mean age was 59-year-old; $53.9 \%$ were women. The most frequently chief complaints were shortness of breath and a combination of fever, cough, and shortness of breath (Table 1).

\section{Test results}

The index test was positive in 41 (78.84\%) subjects with RT-PCR cycle threshold values lower than 30 , and it was negative in $533(91.58 \%)$ subjects with RT-PCR cycle threshold values 30 or greater (Table 2). The overall accuracy was $90.54 \%$.

We found 11 individuals with negative index tests and positive RT-PCR showing median (range) values of cycle threshold values of 25 (21-29), indicating SARSCoV-2 at high concentrations. Ten out of 11 subjects were symptomatic, six had lung infiltrates, and five required hospital admission due to severe symptoms. Among patients with false-negative results, the Panbio COVID-19 antigen testing was carried out at a median of 1.5 days (range 1-7 days) after the onset of symptoms.

There were $49(7.72 \%)$ subjects with false-positive index tests who had no amplification of SARS-CoV-2 gene targets after 45 cycles $(n=48)$ or had a cycle threshold value of 31 ( 1 case). Among the 49 subjects,
25 had symptoms suggesting respiratory tract infection, and 24 were high-risk contacts.

\section{Overall performance of Panbio COVID-19 antigen test}

We estimated the performance characteristics of the index test overall and in symptomatic and high-risk asymptomatic contacts (Table 3). The SARS-COV-2 infection prevalence in our study population was $8.20 \%$, with minor differences between symptomatic (8.47\%) and high-risk asymptomatic subjects (7.66\%). The test's sensitivity was fair, ranging from $72.22 \%$ to $93.75 \%$, and the specificity was high, ranging from $87.05 \%$ to $93.83 \%$, depending on the population studied symptomatic or asymptomatic, respectively (Table 3). Overall, the index test showed a high negative predictive value, between $97.33 \%$ and $99.41 \%$.

\section{Discussion}

We evaluated the performance of the Panbio COVID19 Rapid Antigen Test Device in field conditions in Spain. For a disease prevalence of approximately $8 \%$, the Panbio COVID-19 rapid antigen test showed an acceptable sensitivity of $78.85 \%$ with reasonable specificity of $88.69 \%$ compared with RT-PCR. The test performed better to rule out than in COVID-19 in this population with low disease prevalence. However, 11 (0.20\%) subjects with false-negative Panbio COVID19 rapid antigen test had Ct values of RT-PCT that indicated the possible infectivity of the clinical specimen.

Other field evaluations of the Panbio COVID-19 rapid antigen test have reproduced our performance results. In studies carried out in primary health centers, Albert et al. ${ }^{11}$ showed a sensitivity of $79.6 \%$ with a specificity of $100 \%$, and Bulilete et al. ${ }^{12}$ found similar sensitivity $(71.4 \%)$ and specificity $(99.8 \%)$ for a prevalence of disease around $10 \%$. The study carried out by Albert et al. also showed that in subjects with negative antigen tests and positive RT-PCR, the SARS-CoV-2 could not be cultured from nasopharyngeal samples ${ }^{10}$. In contrast, our study suggests that symptomatic patients with negative Panbio COVID-19 rapid antigen test and positive RT-PCR could transmit the infection. We found that patients had symptoms for $<7$ days, and five required hospitalization due to disease severity. According to SARS-CoV-2 viral dynamics, our patients were in the time frame of maximum risk of contagious infection ${ }^{13}$. Unfortunately, viral culture was not possible at our 
Span J Med. 2021;1(4)

Table 1. Characteristics of the patients included in the study

\begin{tabular}{|c|c|c|c|c|}
\hline \multirow[t]{2}{*}{ Characteristic } & \multicolumn{3}{|c|}{ RT-PCR } & \multirow[t]{2}{*}{$\mathbf{p}$} \\
\hline & $\begin{array}{l}\text { Negative } \\
(\mathrm{n}=582)\end{array}$ & $\begin{array}{l}\text { Positive } \\
(n=52)\end{array}$ & $\begin{array}{c}\text { Total } \\
(n=634)\end{array}$ & \\
\hline Age, mean (SD), years & $59.2 \pm 23.0$ & $57.0 \pm 21.5$ & $59.0 \pm 22.9$ & 0.507 \\
\hline $\begin{array}{l}\text { Age category - no }(\%) \\
<15 \text { years } \\
15-65 \text { years } \\
>65 \text { years }\end{array}$ & $\begin{array}{c}3(0.51) \\
313(53.78) \\
266(45.70)\end{array}$ & $\begin{array}{c}0 \\
34(65.38) \\
18(34.62)\end{array}$ & $\begin{array}{c}3(0.47) \\
347(54.73) \\
284(44.79)\end{array}$ & 0.252 \\
\hline $\begin{array}{l}\text { Sex - no (\%) } \\
\text { Men } \\
\text { Women }\end{array}$ & $\begin{array}{l}271(46.56) \\
311(53.43)\end{array}$ & $\begin{array}{l}21(40.38) \\
31(59.62)\end{array}$ & $\begin{array}{l}292(46.06) \\
342(53.94)\end{array}$ & 0.608 \\
\hline $\begin{array}{l}\text { Chief complaint - no }(\%) \\
\text { Shortness of breath } \\
\text { Fever, cough, and shortness of breath } \\
\text { Fever } \\
\text { General malaise } \\
\text { Cough } \\
\text { Headache } \\
\text { Chest pain } \\
\text { Confusion } \\
\text { Seizures } \\
\text { Diarrhea } \\
\text { Loss of smell } \\
\text { Hemoptysis } \\
\text { Contact with another person with respiratory symptoms }\end{array}$ & $\begin{array}{c}129(22.16) \\
96(16.49) \\
69(11.86) \\
44(7.56) \\
12(2.06) \\
8(1.37) \\
7(1.20) \\
4(0.68) \\
4(0.68) \\
2(0.34) \\
1(0.17) \\
1(0.17) \\
205(35.22)\end{array}$ & $\begin{array}{c}9(17.30) \\
17(32.69) \\
4(6.89) \\
3(5.77) \\
1(1.72) \\
0 \\
0 \\
1 \\
0 \\
0 \\
1(1.72) \\
0 \\
16(30.76)\end{array}$ & $\begin{array}{c}138(21.77) \\
113(17.82) \\
73(11.51) \\
47(7.41) \\
13(2.05) \\
8(1.26) \\
7(1.10) \\
5(0.79) \\
4(0.64) \\
2(0.32) \\
2(0.32) \\
1(0.16) \\
209(32.96)\end{array}$ & $\begin{array}{l}0.429 \\
0.007 \\
0.385 \\
0.685 \\
0.964 \\
0.438 \\
0.486 \\
0.405 \\
0.663 \\
0.815 \\
0.164 \\
0.903 \\
0.528\end{array}$ \\
\hline
\end{tabular}

SD: standard deviation.

Table 2. Pan COVID-19 antigen test results according to the RT-PCR cycle threshold

\begin{tabular}{|c|c|c|c|c|c|}
\hline & \multicolumn{5}{|c|}{ RT-PCR Ct } \\
\hline & $<20(n=14)$ & $20-<25(n=25)$ & $25-<30(n=13)$ & $30-<35(n=4)$ & $\geq 35(n=578)$ \\
\hline Panbio COVID-19 Antigen test positive result & $13(92.86)$ & $21(84)$ & $7(53.85)$ & $1(25)$ & $48(8.31)$ \\
\hline Panbio COVID-19 Antigen test negative result & $1(7.14)$ & $4(16)$ & $6(46.15)$ & $3(75)$ & $530(91.69)$ \\
\hline
\end{tabular}

Ct: cycle threshold.

Table 3. Overall diagnostic performance of Panbio COVID-19 antigen test

\begin{tabular}{|l|c|c|c|}
\hline Statistic, value $(\mathbf{9 5 \%} \mathbf{C I})$ & $\begin{array}{c}\text { All subjects } \\
(\mathbf{n}=\mathbf{6 3 4})\end{array}$ & $\begin{array}{c}\text { Symptomatic subjects } \\
(\mathbf{n}=\mathbf{4 2 5})\end{array}$ & $\begin{array}{c}\text { Asymptomatic high-risk contacts } \\
(\mathbf{n}=\mathbf{2 0 9})\end{array}$ \\
\hline Disease prevalence, \% & $8.20(6.19-10.62)$ & $8.47(6.00-11.53)$ & $7.66(4.44-12.13)$ \\
\hline Sensitivity, \% & $78.85(65.30-88.94)$ & $72.22(54.81-85.80)$ & $93.75(69.77-99.84)$ \\
\hline Specificity, \% & $91.58(89.02-93.71)$ & $93.83(90.96-96.01)$ & $87.05(81.47-91.44)$ \\
\hline Positive likelihood ratio & $9.36(6.92-12.68)$ & $11.71(7.56-8.13)$ & $7.24(4.91-10.66)$ \\
\hline Negative likelihood ratio & $0.23(0.14-0.39)$ & $0.30(0.17-0.50)$ & $0.07(0.01-0.48)$ \\
\hline Positive predictive value, \% & $45.56(38.20-53.11)$ & $52.00(41.16-62.65)$ & $37.50(28.95-46.91)$ \\
\hline Negative predictive value, \% & $97.98(96.63-98.79)$ & $97.33(95.56-98.41)$ & $99.41(96.18-99.91)$ \\
\hline Accuracy, \% & $90.54(87.99-92.70)$ & $92.00(89.00-94.40)$ & $87.56(82.31-91.71)$ \\
\hline
\end{tabular}

$\mathrm{Cl}$ : confidence interval. 
institution to investigate the presence of a viable virus. The SARS-CoV-2 RNA threshold associated with a viable virus in culture is around $>5.9 \log 10$ genome copies $/ \mathrm{mL}$ of the sample, equivalent to an RT-PCR cycle threshold value $<30^{14-16}$.

We also found approximately $8 \%$ of discordant positive index test and negative reference test results, about $50 \%$ of these patients had respiratory tract symptoms. False-positive results can occur if reading tests results are done later than $20 \mathrm{~min}$ or in case of infection by SARS-CoV, which was considered highly unlikely ${ }^{8}$. We cannot provide alternate explanations for these false-positive results.

There is limited information on Panbio COVID-19 rapid antigen test performance to identify SARS-CoV-2 in infected asymptomatic individuals. In our study, we carried out a subanalysis in asymptomatic high-risk subjects. In a total of $16(7.65 \%)$ individuals testing positive by RT-PCR, 15 (93.75\%) yielded positive results with the rapid antigen test. The Panbio COVID19 rapid antigen test's overall sensitivity and specificity were $93.75 \%(95 \% \mathrm{Cl}$; $69.77-99.84 \%)$ and $87.05 \%$ (95\% $\mathrm{Cl}$; 81.47-91.44\%), respectively. Our results showed a better sensitivity value than the reported by Torres et al. (48.1\%) or by Linares et al. (54.5\%) in similar cohorts ${ }^{17,18}$. These differences could lie in the timing of sample collection, or the kinetics of SARS-CoV-2 load in symptomatic and asymptomatic individuals.

A recent meta-analysis including 29 studies and 17,171 COVID-19 subjects evaluated the clinical performance of rapid antigen tests ${ }^{19}$. The overall pooled sensitivity was $78.5 \%$ for symptomatic and $54.5 \%$ for asymptomatic subjects, and the pooled specificity was 99.4\%. The Panbio COVID-19 rapid antigen test showed the highest sensitivity compared to Abbott BinaxNOW'M, Standard ${ }^{\mathrm{TM}}$, and Biocredit ${ }^{\mathrm{TM}}$. Furthermore, the meta-analysis showed that sensitivity was $82.0 \%$ when the symptom onset was $<5$ days but decreased to $75.1 \%$ when the test was used later in the course of the disease. Besides time from symptom onset, another source of heterogeneity was the country of the study. Studies in African and Asian countries showed a decreased sensitivity compared to European and North-American studies, possibly related to a repetitive freeze-thaw process during transportation. The article suggests using kit utilization from local manufacturers to improve the performance of the test. An additional source of heterogeneity was the type of sample collected, being nasopharyngeal swabs the most sensitive compared with saliva.
Among the limitations of our study were the relatively low number of SARS-CoV-2 infections included and lacked precise information regarding the timing of exposure. Therefore, we could not correlate laboratory results with disease courses. However, the study's added value reflects the real-life performance of the Panbio COVID-19 Rapid Antigen Device as a point-of-care test. Due to the lower sensitivity of the Panbio COVID-19 Rapid Antigen Device, RT-PCR would be the preferred diagnostic test. Due to its high specificity, these rapid antigen test results could be helpful to rule out COVID-19. Nevertheless, a small proportion of negative results of the Panbio COVID-19 rapid antigen test can happen in subjects with symptoms in a period of infectivity.

In summary, the performance characteristics of Panbio COVID-19 rapid antigen test indicated that it should not be used as an infection control point-of-care test to decide isolation and cohort of patients. The high transmissibility of new variants of SARS-CoV-2, the lack of a specific treatment, and the potential severity of COVID-19 require a point-of-care test with better performance. Therefore, RT-PCR remains the gold standard for deciding cohorting and isolating patients at risk of SARS-CoV-2 infection.

\section{Funding}

This research did not receive any specific grant from funding agencies in the public, commercial, or not-forprofit sectors.

\section{Conflicts of interest}

The authors declare that there is no conflict of interest.

\section{Ethical disclosures}

Protection of human and animal subjects. The authors declare that no experiments were performed on humans or animals for this study.

Confidentiality of data. The authors declare that they have followed the protocols of their work center on the publication of patient data.

Right to privacy and informed consent. The authors declare that no patient data appear in this article. 


\section{References}

1. Corman VM, Landt O, Kaiser M, Molenkamp R, Meijer A, Chu DK, et al. Detection of 2019 novel coronavirus (2019-nCoV) by real-time RT-PCR Euro Surveill. 2020;25:2000045.

2. World Health Organization. Laboratory Testing for Coronavirus Disease (COVID-19) in Suspected Human Cases: interim Guidance. Geneva: World Health Organization; 2020. Available from: https://apps.who.int/iris/ bitstream/handle/10665/331501/WHO-COVID-19-laboratory-2020.5-eng pdf?sequence=1\&isAllowed=y [Last access on 2021 Jun 26].

3. Centers for Disease Control and Prevention. CDC 2019-Novel Coronavirus (2019-nCoV) Real-Time RT-PCR Diagnostic Panel. Atlanta, Georgia: Centers for Disease Control and Prevention; 2020. Available from: https://www.fda.gov/media/134922/download [Last access on 2021 Jun 26].

4. Dinnes J, Deeks JJ, Adriano A, Berhane S, Davenport C, Dittrich S, et al. Rapid, point-of-care antigen and molecular-based tests for diagnosis of SARS-CoV-2 infection. Cochrane Database Syst Rev. 2020;8:CD013705.

5. Corman VM, Haage VC, Bleicker T, Schmidt ML, Mühlemann B, Zuchowski $\mathrm{M}$, et al. Comparison of seven commercial SARS-CoV-2 rapid point-of-care antigen tests: a single-centre laboratory evaluation study. Lancet Microbe. 2021;2:e311-9.

6. Merino P, Guinea J, Muñoz-Gallego I, González-Donapetry P, Galán JC Antona N, et al. Multicenter evaluation of the Panbio ${ }^{T M}$ COVID-19 rapid antigen-detection test for the diagnosis of SARS-CoV-2 infection. Clin Microbiol Infect 2021;27:758-61.

7. Bossuyt PM, Reitsma JB, Bruns DE, Gatsonis CA, Glasziou PP, Irwig L, et al. STARD 2015: an updated list of essential items for reporting diagnostic accuracy studies. BMJ. 2015;351:h5527.

8. Panbio-COVID-19 Ag Rapid Test Device. Available from: https://dam. abbott.com/en-gb/panbio/120007883-v1-Panbio-COVID-19-Ag-Nasal-AsymptomaticSe.pdf [Last accessed on 2021 Jul 16].

9. Genesig ${ }^{\circledR}$ COVID-19 2G Real-Time PCR Assay. Available from: https:// www.genesig.com/assets/files/Path_COVID_19_CE_IVD_IFU_Issue_3. pdf [Last accessed on $2021 \mathrm{Jul} 16]$.
10. Van Kampen JJ, van de Vijver DA, Fraaij PL, Haagmans BL, Lamers $\mathrm{MM}$, Okba N , et al. Duration and key determinants of infectious virus shedding in hospitalized patients with coronavirus disease-2019 (COVID-19). Nat Commun. 2021:12:267.

11. Albert E, Torres I, Bueno F, Huntley D, Molla E, Fernández-Fuentes MÁ, et al. Field evaluation of a rapid antigen test (Panbio ${ }^{\text {TM }}$ COVID-19 Ag rapid test device) for COVID-19 diagnosis in primary healthcare centres. Clin Microbiol Infect. 2021;27:472.e7-10.

12. Bulilete O, Lorente $P$, Leiva A, Carandell E, Oliver A, Rojo E, et al. Panbio ${ }^{T M}$ rapid antigen test for SARS-CoV-2 has acceptable accuracy in symptomatic patients in primary health care. J Infect. 2021;82:391-8.

13. Sethuraman N, Jeremiah SS, Ryo A. Interpreting diagnostic tests for SARS-CoV-2. JAMA. 2020;323:2249-51.

14. Pekosz A, Parvu V Li M, Andrews JC, Manabe YC, Kodsi S, et al. Antigen-based testing but not real-time polymerase chain reaction correlates with severe acute respiratory syndrome coronavirus 2 viral culture. Clin Infect Dis. 2021;2021:ciaa1706.

15. Gniazdowski V, Morris CP, Wohl S, Mehoke T, Ramakrishnan S, Thielen P, et al. Repeat COVID-19 molecular testing: correlation of SARS-CoV-2 culture with molecular assays and cycle thresholds. Clin Infect Dis. 2020;2020:ciaa1616.

16. Huang CG, Lee KM, Hsiao MJ, Yang SL, Huang PN, Gong YN, et al. Culture-based virus isolation to evaluate potential infectivity of clinical specimens tested for COVID-19. J Clin Microbiol. 2020;58:e01068-20.

17. Torres I, Poujois S, Albert E, Colomina J, Navarro D. Evaluation of a rapid antigen test (Panbio ${ }^{T M}$ COVID-19 Ag rapid test device) for SARSCoV-2 detection in asymptomatic close contacts of COVID-19 patients. Clin Microbiol Infect. 2021;27:636.e1-4.

18. Linares M, Pérez-Tanoira R, Carrero A, Romanyk J, Pérez-García F, Gómez-Herruz $\mathrm{P}$, et al. Panbio antigen rapid test is reliable to diagnose SARS-CoV-2 infection in the first 7 days after the onset of symptoms. $\mathrm{J}$ Clin Virol. 2020;133:104659.

19. Khandker SS, Hashim NH, Deris ZZ, Shueb RH, Islam MA. Diagnostic accuracy of rapid antigen test kits for detecting SARS-CoV-2: a systematic review and meta-analysis of 17,171 suspected COVID-19 patients. $\mathrm{J}$ Clin Med. 2021;10:3493. 